\title{
Detection of Mechanically Deboned Meat in Cold Cuts by Inductively Coupled Plasma-Mass Spectrometry
}

\author{
I. Sarakatsianos ${ }^{1,2}$, N. Manousi ${ }^{3}$, D. Georgantelis ${ }^{4}$, A. Goula ${ }^{5}$ \\ K. Adamopoulos ${ }^{1}$ and V. Samanidou ${ }^{3}$ \\ ${ }^{1}$ Laboratory of Food Industries \& Agricultural Industries Technology, Department of Technologies, \\ School of Chemical Engineering, Faculty of Engineering, Aristotle University of Thessaloniki, GR-54124 \\ Thessaloniki, Greece. \\ ${ }^{2} 3^{\text {rd }}$ Military Veterinary Hospital of Thessaloniki GR 57001, Thermi, Greece. \\ ${ }^{3}$ Laboratory of Analytical Chemistry, Chemistry Department, School of Sciences, Aristotle University of \\ Thessaloniki, GR-54124 Thessaloniki, Greece. \\ ${ }^{4}$ Freelancer, Chemist Ph.D, 25, Th. Sakellaridi, GR-54248, Thessaloniki, Greece. \\ ${ }^{5}$ School of Agriculture, Faculty of Agriculture, Forestry and Natural Environment, Aristotle University of \\ Thessaloniki, Thessaloniki, Greece. \\ *Corresponding Author Email: samanidu@ chem.auth.gr \\ Received 02 October 2018, Revised 28 November 2018, Accepted 29 November 2018
}

\begin{abstract}
In present study the use of Inductively Coupled Plasma-Mass Spectrometry (ICP-MS) was investigated for the detection and differentiation of mechanically deboned meat (MDM) content treated with high pressure techniques in meat products. A number of samples of meat products were prepared containing different proportions of MDM and elemental analysis was performed by a multi-element ICP-MS method after microwave assisted acid digestion of processed meat samples in closed vessels acid microwave digestion method. Element concentrations were plotted versus MDM content and obvious differences were observed for certain elements. Among all examined elements, barium presented a clear trend in correlation of its concentration with the MDM content. The results of this preliminary study indicate that it is possible to detect the proportion of \% MDM content in processed meat products by its correlation to barium concentration.
\end{abstract}

Keywords: Mechanically deboned meat, Meat products, Inductively coupled plasma, Mass spectrometry, Microwave digestion.

\section{Introduction}

Mechanically deboned meat (MDM) is often used as major ingredient in many processed meat products, since it is an important protein source. It is also called mechanically separated meat (MSM) or mechanically recovered meat (MRM) and it can be derived from chicken, hens, broilers, layers, turkey, pork, beef or lamb. However, the most often commercially used is the chicken MDM, which is produced after mechanical removal of the wings, legs, breast, meat and skin [1].
MDM as defined in Regulation (EC) No $853 / 2004$ is obtained by removing meat from flesh-bearing bones, using mechanical means. Carcasses are passed through a mechanical deboner to remove any skeletal muscle still attached to the frames. High pressure is applied to remove the remaining muscle and tissue by forcing it to pass through a series of sieves and/or plates. This procedure results in the production of a finely chopped meat, which can be used in processed meat and poultry products $[2,3]$. 
This results in the loss or the modification of the muscle fiber structure. According to the EUregulation two types of bone separation can be distinguished: "hard separation", which means meat obtained by mechanical means from flesh bearing bones apart from the bones of the head, the extremities of the limbs below the carpal and tarsal joints and, in the case of swine, the coccygeal vertebrae, thus producing high-pressure MDM products and "soft separation", which is the meat obtained mechanically after removal of tendons and connective tissue, thus producing lowpressure MDM products [4,5].

According to the EU Regulation, low- and high-pressure MDM products are defined according to the alteration of bone structure and calcium content. Calcium content is $100 \mathrm{mg} / 100 \mathrm{~g}$ $(1000 \mathrm{ppm})$ as upper limit for low pressure MDM. The pressure used may be different in various machines based on settings applied, however almost all the machines may produce both types by altering the pressure settings [6].

MDM is controlled by legislation in terms of the protein, fat and calcium content, the size and number of solid particles (bone particles) in the deboned meat. The economic impact of the addition of MDM in meat products is significant for the meat products industry. However, there is a risk for human health since MDM is susceptible to deterioration due to the extensive stress and aeration during the machine deboning process and the nature of the product due to its content in bone marrow, heme and lipids [7].

The detection of MDM based on physical properties and chemical composition has been the subject of previous researches. In 2005, Serdaroglu et al. reported on the higher cholesterol and calcium and iron content [8]. Calcium content is an indicator of the amount of bone in meat as reported by several authors [9]. High iron content in mechanically deboned beef and turkey samples is a result of incorporation of red marrow during processing $[10,11]$.

However, till now no correlation has been established between an element content and MDM percentage of the meat product.
In the present study microwave-assisted digestion procedure using closed vessels and nitric acid with hydrogen peroxide has been employed to decompose organic matrix of processed meat samples. Microwave-assisted sample digestion accelerates digestion due to high temperature and pressure and minimizes contamination and losses of volatile elements $[12,13]$.

A multi-element ICP-MS method was used for the determination of various elements in cold cuts samples containing mechanically deboned meat that was treated under low-and high-pressure techniques. However only a few elements could be quantified correlated to the MDM percentage. The preliminary results show a clear trend that has been observed between the MDM percentage of the processed meat products and barium concentration, which was also supported by the analysis of bone, which indicated high percentage in barium. This correlation is reported for the first time, though it can be considered as reasonable as for example for humans, barium is primarily distributed to the bone (almost 90\%). Background levels of barium in human bones are ca $2 \mu \mathrm{g} / \mathrm{g}$ wet weight [14].

\section{Materials and Methods Chemicals and reagents}

All chemicals were of analytical grade. Nitric acid 65\% suprapur was supplied by Merck (Darmstadt, Germany), while hydrogen peroxide $30 \%$ (w/w) was supplied by Sigma-Aldrich (St Louis MO, USA). A multi-element standard solution of $10 \mathrm{mg} / \mathrm{L}$ concentration was purchased from Agilent, Waldbronn, Germany. Ultra pure deionised water with a resistivity of $18.2 \mathrm{M} \Omega \mathrm{cm}$, was obtained from a MembraPure Astacus water purification system (MembraPure $\mathrm{GmbH}$, Bodenheim, Germany).

Meat products were supplied by Creta Farm, a Greek company in the meat and deli meats sector. Different percentages $(w / w)$ of MDM were added in processed meat products: $2 \%, 4 \%, 8 \%, 15 \%, 25 \%, 35 \%$ and $50 \%$. Pure MDM (100\%) as well as pure meat (without mechanical deboning, i.e. $0 \%$ in MDM) were also analyzed. 
Three series of samples were analyzed (Samples 1 and 2 contain high-pressure MDM and sample 3 contains low- pressure MDM). Samples were treated in triplicates and each sample was measured three times. Data were expressed as trace element mean values \pm standard deviation. Correlation of average values was examined versus $\%$ MDM content.

All plastic and glass containers were soaked in $10 \% \mathrm{v} / \mathrm{v} \mathrm{HNO}_{3}$ for $24 \mathrm{~h}$, and then rinsed extensively with ultra pure water prior to use. Plastic containers that came into contact with samples or standards were checked for contamination.

\section{Instrumentation}

A START D Microwave digestion system (Milestone Srl Sorisole (BG) - Italy) was used for sample preparation. Processed meat samples were homogenized by Ultra-Turrax type Yellowline by IKA DI 18 Basic Homogenizer (IKA Werke GmbH \& Co., Staufen, Germany).

Elements were determined using an ICPMS (Agilent 7500s-Agilent Technologies, Waldbronn, Germany). The instrumental settings and operative conditions are reported in Table 1.

Table 1. Instrumental Parameters for the ICP-MS.

\begin{tabular}{lc}
\hline Parameters & Settings \\
\hline Frequency $(\mathrm{MHz})$ & 27.12 \\
Reflect Power (kW) & 1.59 \\
Reflect Matching (V) & 1.66 \\
Sampling depth (mm) & 6.1 \\
Torch-H (mm) & -0.7 \\
Torch-V (mm) & 0.4 \\
Carrier gas (L/min) & 1.19 \\
Nebulizer pump (rps) & 0.1 \\
S/C temperature ( $\left.{ }^{\circ} \mathrm{C}\right)$ & 2 \\
Oxide ions (156/140) & $0.5 \%$ \\
Doubly charged (70/140) & 1.2 \\
Nebulizer type & $\mathrm{Concentric}^{\text {Isotopes }}$ \\
No. of replicates per sample & 3 \\
Quantified elements & $\mathrm{Li}^{6}, \mathrm{Sc}^{45}, \mathrm{Ge}^{72}, \mathrm{Bi}^{205}$ \\
\hline
\end{tabular}

\section{Sample preparation}

EPA method 3052 designed for the "total" analysis in a variety of matrices including soil, sediments, sludge, oils, biological and botanical materials was followed for tissue digestion [15]. Three sample groups of cold cuts were prepared containing different $(\mathrm{w} / \mathrm{w})$ ratios in MDM, namely: 2\%, 4\%, 8\%, 15\%, 25\%, 35\% Kal 50\% w/w. Samples containing 100\% MDM and pure meat with no MDM added were also analyzed for comparison. Two of these sample groups were produced with high pressure MDM and one with low pressure.

All samples were homogenized with UltraTurrax DI-18 disperser (IKA-Werke, Staufen, Germany) at 5,000 rpm for three minutes. For digestion aliquots of $1 \mathrm{~g}$ homogenized sample were accurately weighed using a Teflon vessel. After the addition of $8 \mathrm{~mL}$ of concentrated $\mathrm{HNO}_{3}(65 \%)$ and $2 \mathrm{~mL} \mathrm{H}_{2} \mathrm{O}_{2} 30 \% \mathrm{w} / \mathrm{w}$, the digestion vessel was closed and heated in the microwave digestion system. The temperature was increased gradually up to $200^{\circ} \mathrm{C}$ in $10 \mathrm{~min}$ and remained constant for another $10 \mathrm{~min}$.

The obtained solutions were allowed to cool at room temperature and quantitatively transferred into a glass volumetric flask of $50 \mathrm{~mL}$ (class A) and completed to volume with ultrapure deionised water. Analysis was performed by ICPMS, following external calibration. Filtration was not necessary since the resultant digestate was clear enough.

At regular intervals during analysis, calibration standards were analysed to monitor instrument performance. Additionally, ultrapure deionised water blanks were frequently checked for cross contamination. Blanks were prepared by following the whole analytical procedure without samples. Measured concentration in the digested sample expressed in $\mu \mathrm{g} / \mathrm{L}$ as calculated by calibration curve is converted to concentration in tissue expressed in $\mu \mathrm{g} / \mathrm{kg}$ by the following equation:

$\mathrm{C}_{\text {digested sample }} /(20 \times \mathrm{m})=\mathrm{C}_{\text {tissue }}$, where $\mathrm{m}$ is the mass of MDM in grams. 


\section{Standard solutions}

All calibration standard solutions were prepared from $10 \mathrm{mg} / \mathrm{L}$ multi-element standard solution by sequential dilution with $5 \%(\mathrm{w} / \mathrm{w})$ $\mathrm{HNO}_{3}$ in ultrapure deionised water.

All standards, reagent solutions and samples were kept in plastic containers and stored in the refrigerator before measurements. The working standard solutions were freshly prepared daily prior to the analyses and the concentrations were $0,10,20$ and $50 \mu \mathrm{g} / \mathrm{L}$ for $\mathrm{Mn}, 0,20,100$ and $200 \mu \mathrm{g} / \mathrm{L}$ for $\mathrm{Zn}, 0,2,10$ and $20 \mu \mathrm{g} / \mathrm{L}$ for $\mathrm{Sn}$ and 0 , 10,50 and $100 \mu \mathrm{g} / \mathrm{L}$ for $\mathrm{Ba}$.

\section{Results and Discussion Method calibration}

For the quantitative analysis, external calibration technique was used. The calibration curves were constructed at four concentration levels. The correlation coefficients for all the calibration curves were at least 0.9999 , showing good linear relationship throughout the ranges of concentrations studied. For checking the absence of polyatomic interferences several isotopes of the elements were measured and the isotopic ratio in the digested solution of the samples was examined.

\section{Statistical analysis- Correlation of elements concentration with \% MDM content}

Regression analysis was applied to the results. Concentration of different elements was plotted versus \% MDM content. From the examined elements, only barium showed to follow the linear model, since an obvious trend of linear relationship is observed in all group samples. Manganese showed also a linear relationship in samples 1 and 2, which contain high-pressure MDM and no correlation in sample 3, which contains low-pressure MDM. This difference can be attributed to the processing technology. Fig. 1-4 illustrate the results for selected elements. The threshold for the safe quantification of MDM is $4 \%$.
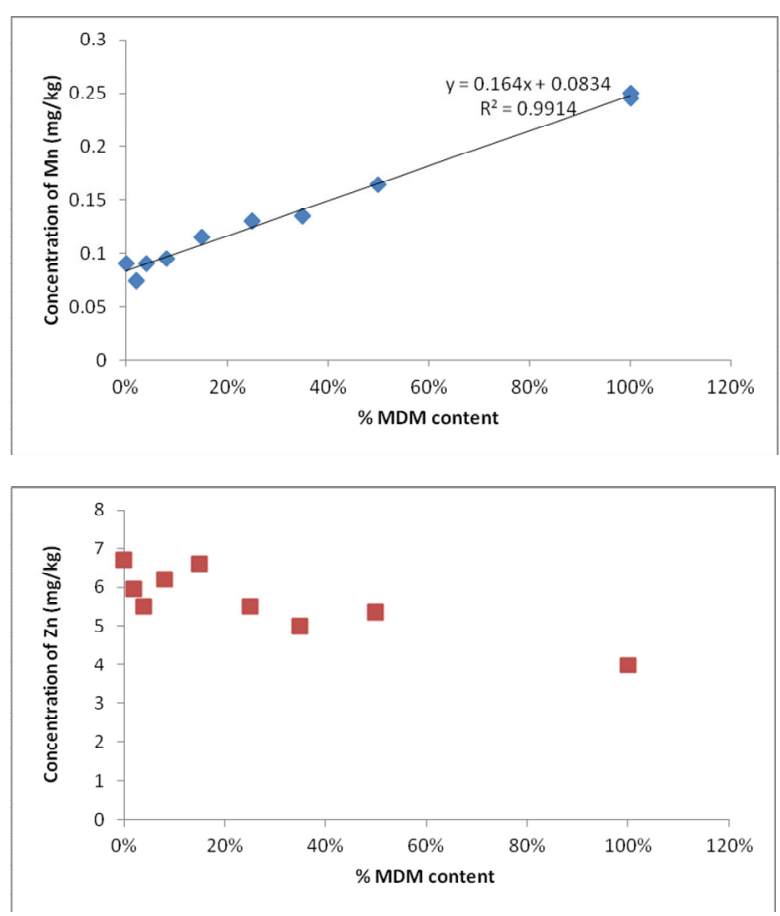

Figure 1. Relationship of concentration for $\mathrm{Mn}$ and $\mathrm{Zn}$ versus \% MDM content for Group Sample 1 (Cold cuts containing highpressure MDM)
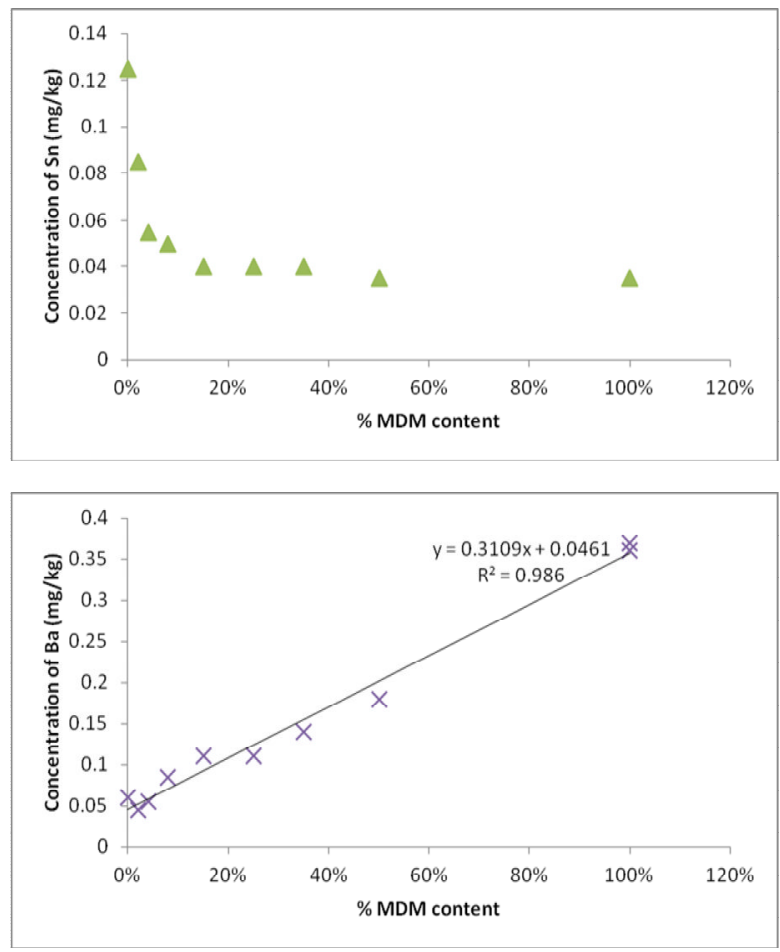

Figure 2. Relationship of concentration for $\mathrm{Sn}$ and Ba versus \% MDM content for Group Sample 1 (Cold cuts containing highpressure MDM) 

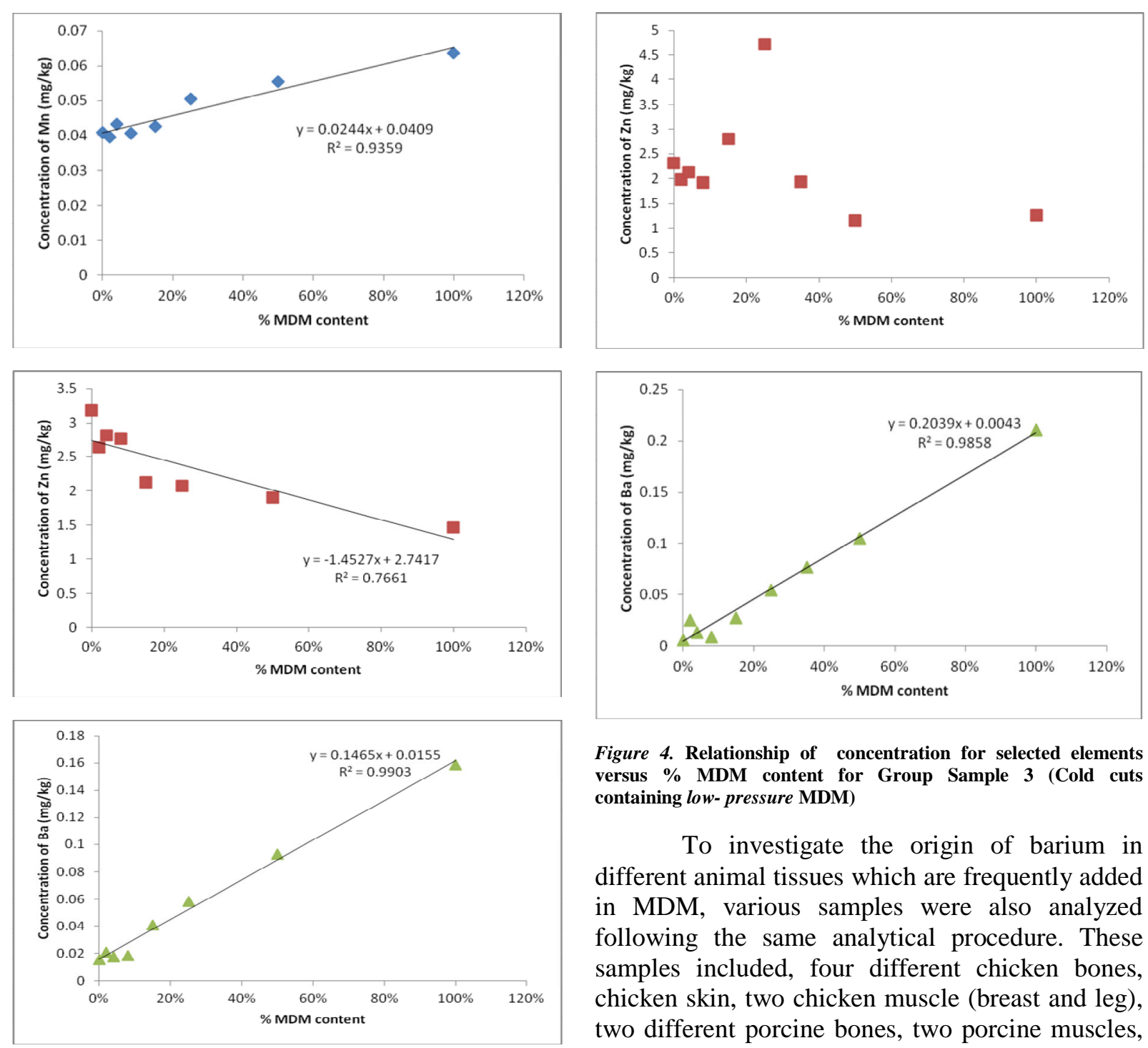

Figure 3. Relationship of concentration for selected elements versus \% MDM content for Group Sample 2 (Cold cuts containing high- pressure MDM).

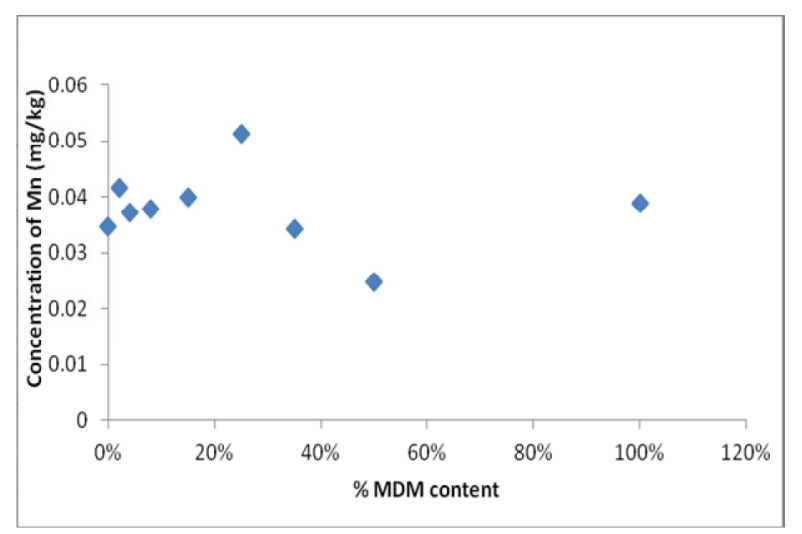

Figure 4. Relationship of concentration for selected elements versus \% MDM content for Group Sample 3 (Cold cuts containing low-pressure MDM)

To investigate the origin of barium in different animal tissues which are frequently added in MDM, various samples were also analyzed following the same analytical procedure. These samples included, four different chicken bones, chicken skin, two chicken muscle (breast and leg), two different porcine bones, two porcine muscles, porcine marrow, porcine periosteum and porcine vessel, as well as four commercial products (paste and parizer) for comparison.

Considering that MDM contains an increased proportion of bone particles (whose main element is calcium), the correlation found between barium content and MDM percentage may be explained by the increased concentration of barium, as this element is primarily distributed to the bone according to the literature, at least in humans [14]. These two chemical elements belong to the same group in periodic table and they exhibit similar behavior as they possess the same chemical properties. Fig. 5 shows the results of barium concentration in various animal tissues. 


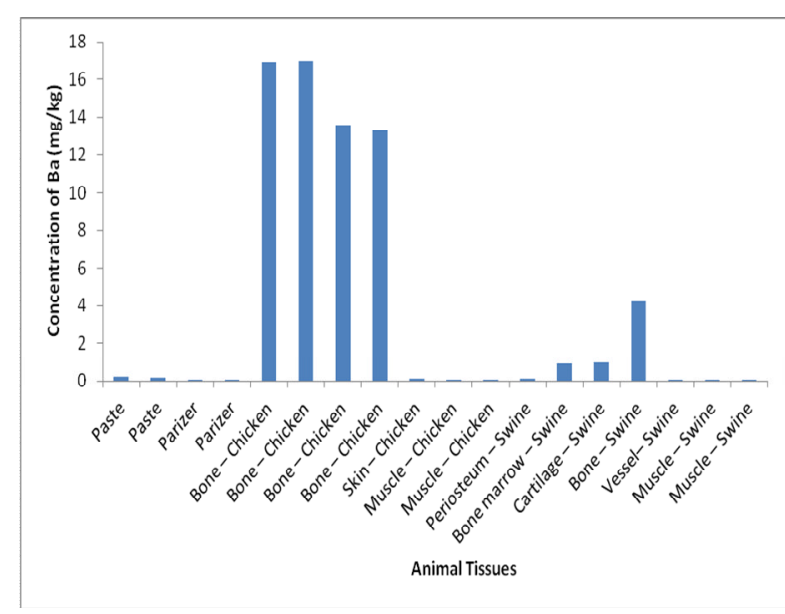

Figure 5. Barium concentration in various animal tissues.

\section{Conclusion}

This preliminary study which described herein shows that we can predict the \% MDM content in processed cold cuts by determining the concentration of barium, which can be attributed to the fact that barium is mainly distributed to the bone. The analysis of various bone types showed that they contain considerable amounts of barium. The fact that MDM contains bone particles leads to the correlation of barium and MDM percentage. Regression analysis of the findings of current research shows that quantification is achieved when MDM content is higher than $4 \%$. More experiments need to be carried out, in order to create a statistical model for the prediction and calculation of a more accurate and sensitive \% MDM calculation. Thus, barium content can be used as quality indicator of processed meat products in meat and deli meats factories. Manganese showed also a linear relationship in containing high pressure MDM and no correlation in containing low pressure MDM. This difference can be attributed to the processing technology.

\section{Acknowledgments}

The authors wish to thank Creta Farm for the kind donation of processed meat products.

\section{Declaration of Interest}

The authors declare no conflict of interest.

\section{References}

1. L. Day and H. Brown, Meat Sci., 58 (2001) 31.

https://doi.org/10.1016/s03091740(00)00127-3 [accessed September 2018].

2. Regulation (EC) No 853/2004 of the European Parliament and of the Council of 29 April 2004 laying down specific hygiene rules for food of animal origin. https://eurlex.europa.eu/LexUriServ/LexUriServ.do?uri =OJ:L:2004:139:0055:0205:EN:PDF

3. G. J. Mountney and C. R. Parkhurst. Poultry Products Technology. Food Products Press, New York, (1995).

4. http://agris.fao.org/agrissearch/search.do?recordID=IT1998061463 [accessed September 2018].

5. http://ec.europa.eu/food/fs/sc/oldcomm4/out 16 en.html [accessed September 2018].

6. http://www.wpsa.com/index.php/publications /wpsa-proceedings/2013/xxi-europeansymposium-on-the-quality-of-poultry-meatand-the-xv-european-symposium-on-thequality-of-eggs-and-egg-products/1230mechanically-separated-meat-msm-efsaassessment-on-public-health-risks-andparameters-for-classification/file [accessed September 2018]

7. K. E. Moerck and H. R. Ball, J. Food Sci. 39 (1974) 876.

https://doi.org/10.1111/j.13652621.1974.tb07265.x

8. M. Serdaroğlu, G. T. Yildiz, and N. Bağdatlioğlu, Turk. J. Vet. Anim. Sci., 29 (2005) 797. http://journals.tubitak.gov.tr/veterinary/issues /vet-05-29-3/vet-29-3-32-0402-19.pdf

9. A. R. Crosland, R. L. S. Patterson, R. C. Higman, C. A. Stewart and K. D. Hargin, Meat Sci., 40 (1995) 289. https://doi.org/10.1016/03091740(94)00060-K

10. B. P. Demos and R. W. Mandigo, J. Food. Sci., 60 (1995) 576.

https://doi.org/10.1111/j.13652621.1995.tb09830.x 
11. G. Strmiskova, F. Strmiska and J. Dubravicky, Die Nahrung, 37 (1993) 94. https://doi.org/ 10.1002/food.199303701222a

12. J. T. P. Barbosa, C. M M. Santos, V. N. Peralva, E. M. M. Flores, M. Korn, J. A. Nóbrega and M. G. A. Korn, Food Chem., 175 (2015) 212.

https://doi.org/ 10.1016/j.foodchem.2014.11.092

13. H. M. Kingston, P. J. Walter In: Inductively Coupled Mass Spectrometry, A. Montaser, Wiley-VCH, NY (1997) 33. https://books.google.com.pk/books?hl=en\&lr $=$ \&id $=-$

c9nIF gc30C\&oi=fnd\&pg=PR19\&dq=H.+M +Kingston, ++ Inductively+Coupled+Mass $+\mathrm{S}$ pectrometry,+A.+Montaser\&ots=ffu24CTAiI \&sig=NrbQtc1YNWYw_Fpvoldyc52QI20\#v =onepage \&q=H.\%20M.\%20Kingston $\% 2 \mathrm{C} \%$ 20\%20Inductively\%20Coupled\%20Mass $\% 2$ oSpectrometry\%2C\%20A.\%20Montaser \&f= false.
14. H. A. Schroeder, I. H. Tipton and A. P. Nason, J. Clinical Epid., 25 (1972) 491. https://doi.org/10.1016/00219681(72)90150-6. 